\title{
Anesthesia and Apoptosis in the Developing Brain: An Update
}

\author{
Andrew Davidson · Jurgen C. deGraaff
}

Published online: 23 December 2012

(C) Springer Science + Business Media New York 2012

\begin{abstract}
There is an increasing concern regarding the risk of anaesthetic neurotoxicity in children. Numerous animal and in vitro studies have demonstrated that general anaesthetics cause a variety of potentially harmful effects on the developing brain. An increased level of neuronal apoptosis is one such effect. In some studies, exposed animals have experienced long-lasting neurobehavioural changes. These effects occur at very specific stages of development and are dose-dependent. There are several plausible mechanisms for these deleterious effects; however, the exact mechanism remains unclear. Most importantly, histological and behavioral effects have been demonstrated in non-human primates. Human research evaluating the long-term effects of anaesthesia on brain development is limited and, to date, has been limited to retrospective observational cohort studies. The results of these studies have not been consistent, with some demonstrating an association between surgery and adverse neurobehavioral outcome and others showing no association. Furthermore, these cohort studies have numerous weaknesses and limitations. An ongoing randomized clinical trial aims to provide clear evidence regarding the clinical safety of anesthetics up to $1 \mathrm{~h}$ in neonates and older children.
\end{abstract}

\footnotetext{
A. Davidson $(\bowtie)$

Department of Anaesthesia and Pain Management, Royal Children's Hospital \& Murdoch Childrens Research Institute, Flemington Road, Parkville, Melbourne, VIC 3052, Australia e-mail: andrew.davidson@rch.org.au

J. C. deGraaff

Department of Anesthesiology, Wilhelmina Children's Hospital, University Medical Centre Utrecht, Mailstop Q 04.2.302, PO Box 85500, 3508 GA Utrecht, The Netherlands e-mail: j.c.degraaff-2@umcutrecht.nl
}

Keywords Pediatric anesthesia - Neurotoxicity . Apoptosis

\section{Introduction}

Numerous studies in animals have shown that general anaesthetic agents can cause apoptosis and other changes to neurons in the developing brain [1]. Several plausible mechanisms have been proposed to explain these effects. However, translating animal data to humans is fraught with problems, and the clinical relevance of these findings remains uncertain. In human clinical studies, there is mixed evidence for an association between exposure to anaesthesia in childhood and subsequent adverse neurobehavioral outcome. There are many limitations to these studies, which may explain the contradictory findings. At this stage, it is impossible to definitively know whether early exposure to anaesthesia holds any clinically relevant long-term risk for the brain.

\section{Normal Development}

The human brain undergoes a relatively long and complex development. The human brain doubles its size in the first year of life, and reaches $90 \%$ of its maximum size by 6 years of age. The stages of development are: neurogenesis and proliferation, differentiation, synapse formation, and myelination. In some respects, the brain continues to develop throughout life; however, the rate of change is greatest in the fetus and early childhood. There are many trophic factors and receptors involved in development. GABA and NMDA are two of the receptors involved in normal development. Crucially, the morphology and 
function of receptors involved in development changes with age. This explains why particular drug effects may be observed during specific developmental periods.

The numbers of synapses and neurons halves during development. The number of synapses is greatest during infancy and decreases throughout childhood. The number of neurons is greatest in the developing fetus but decreases through the neonatal period and infancy. The loss of neurons occurs via apoptosis. Apoptosis, or cell suicide, is an organized energy-consuming process by which "unwanted" cells are removed from the organ. This contrasts with the more destructive "excitatory" process of cell death. Apoptosis involves chromatin aggregation, the condensation of cellular organelles, and the development of apoptotic bodies that are readily consumed by phagocytosis. Apoptosis may be triggered via two pathways: intrinsic and extrinsic. Both pathways, however, share a final common sequence that identifies an important marker of apoptosis: activation of the enzyme caspase 3 [2]. Many factors can trigger apoptosis including normal growth and development, diseases, and anesthetics. Apoptosis is the key to normal organ remodeling that occurs in all multicellular organisms. It explains how tadpoles lose their tails and how humans lose the webs between their fingers. Thus, the effects of other triggers on apoptosis must be understood in the context of the normal rate of apoptosis in the developing newborn.

Anaesthetics act on GABA and NMDA receptors. GABA and NMDA receptors are directly involved in cell proliferation, migration, cell survival, and dendritic maturation. The GABA and NMDA receptors are indirectly involved in the balance of activity and, thus, the generation of trophic factors that drive differentiation, growth, and apoptosis.

\section{Effects Seen in Animal Studies}

It is well known that the developing brain is vulnerable to external influences. Exposure to alcohol and antiepileptic drugs causes neurodevelopmental disorders such as the fetal alcohol syndrome. During the investigation of these exposures, it was noted that GABA agonists and NMDA antagonists can trigger accelerated apoptosis in the young rodent brain. In fact, blockade of NMDA receptors with MK801 resulted in widespread apoptosis in the brains of 7-day-old rats [3]. Subsequently, many other studies demonstrated accelerated neuronal apoptosis in newborn rodents after they were exposed only to general anaesthetics such as ketamine, propofol, nitrous oxide, sevoflurane, isoflurane, desflurane, and benzodiazepines, without any other insults [2, 4-10]. In rats, the effect of anesthetics on apoptosis was greatest in 5-7 day olds. Furthermore, more prolonged exposure to anesthetics as well as exposure to multiple anaesthetic agents in combination exacerbated the severity of the apoptosis.
Some studies have also demonstrated long-term cognitive and learning defects in the exposed animals, although this has not been a consistent finding [4, 10-12]. Interestingly, $4 \mathrm{~h}$ of $1 \mathrm{MAC}$ of sevoflurane caused significant memory problems in rats; however, if they were housed in an enriched environment during their subsequent development, the memory performance was significantly better than those kept in normal cages in both exposed and nonexposed rats, to the extent that no difference could be detected between those exposed and those not exposed to anesthetics [13•]. This demonstrated that neurobehavioral development is multifactorial, and that anaesthesia exposure is likely just one of many important influences, some of which may be far greater than the anaesthesia exposure.

Most of the animal data were derived from rodents; however, data are also emerging in the non-human primate model. Increased apoptosis has been observed in monkeys during late gestation or at 5 days postnatal age after $24 \mathrm{~h}$ of ketamine, although no effect was observed in 35-day-old monkeys. Interestingly, the severity of the apoptosis after $3 \mathrm{~h}$ of ketamine in vulnerable monkeys was similar to that in the control group [14], whereas in another study the severity after $5 \mathrm{~h}$ was significantly increased [15••]. In followup studies, persistent learning deficits were documented in 5-day-old monkeys after $24 \mathrm{~h}$ of ketamine [16 $\left.{ }^{\circ}\right]$. In these and other studies, the doses of ketamine that were used $(20-50 \mathrm{mg} / \mathrm{kg} / \mathrm{h})$ were much greater than those used to anesthetize humans $(5-10 \mathrm{mg} / \mathrm{kg} / \mathrm{h})$. It is important to understand that monkeys require relatively large doses of ketamine for any clinical effect. In these studies, ketamine caused more apoptosis in the fetus than in the neonatal animal. Isoflurane has been shown to cause more apoptosis in the newborn monkey than ketamine. As might be expected, apoptosis increased after $5 \mathrm{~h}$ of isoflurane [17•0]. These studies also found that accelerated apoptosis occurred in different areas of the brain when animals of differing ages were exposed to different anesthetics. This suggests that the clinical effects of anesthetics may vary with the age at the time of exposure and the anesthetic agent.

Although most general anesthetics have been shown to cause apoptosis, two anesthetics, Xenon and dexmedetomidine, are the exceptions to date $[18,19]$.

Foetal and neonatal exposure to opioids, and in particular chronic exposure to opioids, have been associated with a variety of neuronal changes [20]. There are very few, if any, studies that have specifically examined whether or not short exposure to opioids results in apoptosis.

\section{Mechanism of Anaesthesia-Induced Apoptosis}

The exact mechanism by which anesthesia induces apoptosis is unknown. One theory holds that anesthesia causes 
relative inactivity that results in a loss of trophic factors, which in turn triggers apoptosis. This theory fits with the "use it or lose it" notion that we are born with an excess of neurons and synapses and that we trim those that are not active. There are some data to support this mechanism. Active axons normally release tPA and proBDNF [21]. The tPA cleaves plasmin to plasminogen which in turn converts proBDNF to BDNF. BDNF acts at the TrkB receptor on the dendrite to promote cell survival. If the axon is not active, proBDNF but not tPA, continues to be released. Without tPA, there is no plasminogen, and thus proBDNF is not converted to BDNF. The proBDNF acts on the $\mathrm{p} 75^{\mathrm{NTR}}$ receptor on the dendrite, which results in cell death.

It is possible that anesthesia induces inactivity via GABA receptors. However, against this argument, GABA receptors are excitatory at this phase of neurodevelopment. Also, giving a GABA antagonist with an anesthetic does not reduce the effect. If inactivity is the mechanism, then it is not simply via GABA activation [19]. This is reinforced by the observation that apoptosis occurs with anesthetics such as ketamine, even though they have no GABA activity.

Another suggested mechanism is based on the fact that glutamate may cause excitotoxic cell death via NMDA receptors. It has been suggested that anesthetic-induced NMDA blockade leads to acute upregulation of the NMDA receptor, and that when the anesthetic is removed, there is an increased risk of subsequent excitotoxic neurotoxicity via the upregulated NMDA receptors [22]. However, this argument is undermined by the observation that apoptosis occurs with agents that have no action on NMDA receptors (such as propofol), and also that apoptosis does not occur with agents such as Xenon which are known to be NMDA antagonists. It has also been observed that cell death can occur during the period of NMDA blockade. Lastly, both racemic and s-ketamine have similar degrees of toxicity [23].

\section{Effects Other than Apoptosis}

General anesthetics have effects on the newborn brain apart from apoptosis. Propofol and inhalational anesthetics cause changes in dendritic spine morphology [24•, 25-27]. In 5- and 10-day-old animals, anesthetics cause a loss of dendritic spines, while in 15-, 20-, and 30-day-old animals, it increases the number of dendritic spines. Changes in the neuronal cytoskeleton have also been observed, possibly via the $\mathrm{p} 75^{\mathrm{NTR}}$ receptor and subsequent RhoA activation [28]. Altered neurogenesis and abnormal re-entry into the cell cycle has also been observed [29, 30]. Lastly, anesthetics have been shown to impair mitochondrial function [31]. It is unknown if all these observed effects are linked with a common mechanism.

\section{Translating Animal Data to Humans}

It is difficult to appreciate the relevance of data obtained from animals to clinical scenarios in humans. There are many factors that make the translation of findings difficult. First, the age of exposure is difficult to interpret. A 5- to 7-day-old rat may correspond to a late trimester human foetus or a neonate, but this is by no means certain. Although apoptosis may be observed in 7-day-old rats, other dendritic changes may be observed over a far wider age in rodents. It is also difficult to translate the duration of exposure. Brain develops rapidly in a rodent, over a brief time frame. Several hours in the life of a rat may be synonymous with several weeks of life in a human. A toxic stimulus for what is a significant amount of time relative to the time required for a rat to develop may not be analogous to a toxic stimulus for a relatively brief period in the human. However, the difference between the anesthetic effects in rodents and humans is further complicated by the fact that the human brain is far more complex, and an injury during crucial periods of development may be far more significant, in the human. Alternatively, the human brain may have a greater capability of recovering after an injury. These issues remain unresolved.

Translating the neurobehavioral effects is also difficult. It is impossible to determine fine changes in behavioral domains in a rodent. Drug doses in rodents often differ from those in humans. Although the doses of inhalational anesthetics are similar across species, the doses of intravenous anesthetics in animals are much greater than in humans. Lastly, most experiments that were conducted in newborn rodents were not performed during a surgical stimulation or in the presence of inflammation. Some studies have reported no effect of surgical stimulation on apoptosis in the rodent model [13•], whereas others found that it attenuated the response [32]. This issue remains unresolved.

Infant rodents are also challenging to anesthetize. The experiments have a very high mortality. It is impossible to know if the oxygen saturation and cerebral perfusion in these experiments were optimal. In summary, the animal data that demonstrate increased apoptosis are concerning and the experiments are crucial if we are to understand the mechanisms and possible therapies to reduce anesthesiaassociated apoptosis. Human studies are needed to determine the relevance of these phenomena in the human clinical situations.

\section{Clinical Evidence}

Clinical research evaluated the effects of anesthesia on the developing brain, although the evidence is scarce and 
limited to retrospective observational cohort studies. Unfortunately, there are many limitations inherent in these studies including the influence of confounding variables, the difficulty in gathering detailed information about the surgery and anesthesia (exposure) and the difficulty defining the optimal neurobehavioral domain for follow-up (outcome).

The paediatric and neonatology literature is replete with evidence linking major surgery in infancy with an increased risk of adverse neurobehavioral outcome. Furthermore, children who underwent cardiac surgery or repair of esophageal atresia [33], preterm infants who underwent a laparotomy or hernia repair $[34,35]$ as well as prematurity and sepsis themselves, increase the risk of poor neurobehavioral outcomes. Children with major malformations are at greater risk for chromosomal abnormalities. In many of these reports, a poor outcome may have resulted from a panoply of confounding variables, in addition to the anesthetic. The precise role of anesthesia in these complex cases is very difficult to tease out from among the multitude of variables. Neonatologists have also reported that undergoing even a minor surgical procedure increases the risk of a poor neurobehavioral outcome in otherwise healthy children [36, 37].

More recently, several cohort studies assessed the risk of anesthesia in terms of neurobehavioral outcomes. In the first analysis, the presence of learning disorders using the local school system and the Reading Center/Dyslexia Institute was identified in 593 children from a retrospective birth cohort of 5,357 children from Olmsted County (Minnesota, USA), who were born between 1976 and 1982 and who received a general anesthetic before 4 years of age $[38 \bullet, 39,40,41 \cdot, 42]$. Using regression analyses to calculate hazard ratios (HRs) for anesthetic exposure as a predictor for learning disorders with adjustment for several confounding factors (gestational age at birth, sex, and birth weight) [41•], the authors found that a single exposure to anesthesia before 4 years of age was not associated with an increased risk for developing learning disorders [HR, 1.0; $95 \%$ confidence interval (CI): 0.79-1.27]. However, multiple exposures to anesthetics increased the risk of learning disorders such that the HR for two anesthetics was 1.59 (95\% CI: 1.06-2.37) and for three of more anesthetics was 2.60 (95\% CI: 1.60-4.24). Furthermore, the risk for learning disorders was associated with a greater cumulative duration of anesthesia. However, this analysis suffered from several limitations. Other factors associated with learning disorders, such as the presence of syndromes and chronic diseases, are more likely to require multiple anesthesia. The authors attempted to correct for these factors by adjusting for the American Society of Anesthesiologist physical status, although this did not alter the results significantly. Additional confounding factors that may have affected the regression that were not included are family, genetic factors, and social economic status. Furthermore, $90 \%$ of the children received halothane and nitrous oxide anesthesia, without monitors such as pulse oximetry and capnography, which are considered to be standards monitors today. These differences in practice since 1982 limit the generalizability of these studies to present clinical practice.

Three other analyses of the same population have been published [38• 40, 42]. To reduce the influence of confounders, 350 children who were exposed to anesthesia before 2 years of age were matched with 700 children who had not been exposed to anesthesia on the basis of risk factors that are known to influence the incidence of learning disorders (birth weight, gender, mother's education, gestational age, birth date \pm 12 months) [38•]. Again, the exposure to multiple anesthetics (but not to a single exposure) was associated with and increased the risk of developing learning disorders (HR, 2.12; $95 \%$ CI: 1.26-3.54) and a decrement in cognitive, memory subscale, and achievement in mathematics (California Achievement Test and Test of Cognitive Skills). The review identified an association between exposure to anaesthesia before 2 years of age and the development of attention deficit/hyperactivity disorder (ADHD) in the same population [42]. After adjusting for confounders, multiple exposures (HR, 1.95; $95 \%$ CI: 1.03-3.71), but not single exposures (HR, 1.18; $95 \%$ CI: 0.79-1.77) to anesthesia, were associated with an increased risk for ADHD. Many of the limitations in their initial study also apply to these later studies.

In a retrospective analysis of a birth cohort from 1999 to 2002 that were enrolled the New York Medicaid Program, the authors sought an association between anesthesia for inguinal hernia repair and the risk of developing behavioral and developmental disorders [43•,44]. They compared 383 children who had hernia repair before 3 years of age with a sample of 5,050 age-matched children without a history of hernia repair [43•]. After correcting for age, sex, and complicating birth-related conditions (such as low birth weight, perinatal hypoxia), children who had hernia repair were more than twice as likely to be diagnosed with a developmental or behavioral disorder (HR, 2.3; $95 \% \mathrm{CI}$ : 1.3-4.1).

To reduce the influence of confounders such as age, home environment, parenting style, educational systems, and neighborhood or ecological characters, researchers performed a nested case control study with twin siblings of the same database. Exposure was defined as anesthesia before 3 years of age and [44]. Amongst 10,450 twin siblings, there were only 138 twin siblings discordant for exposure. Amongst the 138, development or behavioral disorders were recorded in 11 siblings unexposed and 9 that were exposed, giving an odds ratio for adverse 
outcome of 0.9 (95\% CI: 0.6-1.4). Unfortunately, these numbers are too small to draw any conclusions. Looking at the entire twin sibling population, they found an increased risk for development and behavioral disorders when the children were exposed to anesthesia (HR, 1.6; $95 \%$ CI: 1.4-1.8); increasing from 1.1 (95\% CI: $0.8-1.4)$ for a single exposure to 2.9 (95\% CI: $2.5-3.1$ ) for two exposures and to 4.0 (95\% CI: 3.5-4.5) for three or more operations. However, both these studies have serious limitations. In both studies, the researchers used the administrative data of a health insurance with ICD-9 codes to determine exposure and outcome. The diagnosis of ADHD, and development and behavioral disorders may be affected by misclassification and underreporting. Moreover, Medicaid is a health insurance program for members of the lowest socioeconomic status in New York State, a population that is more likely to suffer from mental illness, reducing the generalizability of the results.

Academic performance was evaluated in 2,689 children who had hernia repair in infancy and who were compared with a randomly selected age-matched group of 14.575 children from a Danish birth cohort from 1986 to 1990 [45••]. Those who were exposed to surgery scored worse than the control group (average score 0.26 lower; $95 \%$ CI: 0.21-0.31). However, after correcting for sex, birth weight, parental and maternal age, and education, there was no difference in the academic performance between those exposed and unexposed to anesthesia $(-0.04,95 \% \mathrm{CI}$ : $-z 0.09$ to 0.01 ). In a smaller but similar study using school records in the state of Iowa, USA, researchers examined children who had undergone surgery in infancy and who had no other risk factors for neurobehavioral delay [46]. The mean of their school test scores was similar to the population average; however, the exposed cohort did have a higher percentage of children who scored below the fifth centile.

As mentioned, the main limitation of these retrospective observational studies is the influence of known and unknown confounders. An option to correct for confounders such as education level of parents, socioeconomic status, and environmental and genetic factors is cohort research in monozygotic twins. In a study of monozygotic births in which the twin pairs were exposed to anesthesia before 3 years of age, those who received anesthesia scored lower on educational achievement and had more cognitive problems than those who were not exposed to anaesthesia [47••]. However, there was no difference within twin pairs who were discordant for anesthetic exposure. This suggests that anesthesia at early age may be a marker for a child's vulnerability to develop later learning problems, although anesthesia per se does not appear to be a risk. However, this study also has some limitations. Details about the kind of surgery and anesthesia were omitted. It is possible that anesthesia was too brief to influence cognitive function. These data highlight several weaknesses in this area of research because neither the age at the time of exposure nor the duration of exposure to anesthesia has been firmly established. Cohort studies that include broad age ranges may not be relevant to anesthetic exposure in only the neonate. Similarly, failure to find an increased risk of neurocognitive dysfunction after a brief exposure to anesthesia does not preclude an increased risk after a greater exposure. Another limitation in the cohort studies is which neurobehavioral outcome should be studied? Examining broad composite scores such as school performance may miss deficits in sub-domains of neurobehavioral activity. However, performing multiple tests is logistically difficult and assessing multiple tests increases the risk of a Type I statistical error. A study in West Australia used an established pregnancy cohort (the Raine cohort), which included 2,608 children who had extensive neurobehavioral testing by 10 years of age [48]. The 321 children who were anesthetized before the age of 3 years were more likely to have problems in receptive and expressive language and cognition compared with those who had not been anesthetized. This study may be of limited relevance since many of the children who received anesthesia only had ear tube surgery. This surgery usually lasts less than $5 \mathrm{~min}$ and is often performed in children who are at increased risk for language delay.

\section{Future Clinical Studies}

In order to reduce the influence of confounding variables, an ambi-directional sibling matched cohort study is currently enrolling patients. Approximately 500 ASAs 1 and 2 physical status children will receive a single general anesthetic for inguinal hernia surgery before 3 years of age. They will be matched with an unexposed sibling from a retrospective database. Participants will be evaluated prospectively with an extensive neuropsychological battery between age 8 and 15 years [49]. The scale of the study, the presence of detailed information about anesthesia, and the extensive test battery will allow detailed analyses of the relationship between timing of anesthesia and neurocognitive deviation.

Ideally, to determine the influence of anesthesia, without the confounding effect of surgery and pathology, on neurocognitive development, one should randomize children between anesthesia and no anesthesia, with or without surgery. Such a factorial design is impossible because of ethical considerations. A randomized clinical trial that compares general anesthesia with regional (spinal) anesthesia (the GAS study) is one plausible alternative [50]. However, such prospective trials are expensive to complete and slow to generate results. The results of this study will 
only be available when all the children have been recruited and followed for up to 5 years. One criticism of this study is that, since hernia repair is a minor procedure, the results of the GAS study may only be generalizable to anesthesia for minor procedures that are limited to $1 \mathrm{~h}$. However, since the majority of procedures in children last less than 1 $\mathrm{h}$, the results of this study may be very important in establishing some metrics for the safety of anesthesia in the majority of children who undergo surgery.

\section{Conclusion}

Given the problems thus far of translating the animal data to humans and the weaknesses in the clinical studies of neurocognitive dysfunction in children who were anesthetized at a young age, it is difficult to speculate how such research should affect clinical pediatric practice. Pediatric anesthesia societies and regulatory authorities agree that the published animal data are concerning and that exposure to anesthesia may be related to poor outcomes in certain circumstances. One federal agency has called for a moratorium on elective surgery in children less than 3 years of age, even though they concede there is no causality between administering anesthetics and neurocognitive dysfunction in humans [50]. Evidence has shown that stress and pain cause neurobehavioral changes in children. Withholding anesthesia or analgesia may exacerbate these changes. Similarly, children rarely undergo totally elective procedures, so that delaying surgery may raise the risk of complications from the uncorrected surgical defect. Finally, the evidence is very clear that anesthesia in infants less than 1 year of age carries with it a high risk of morbidity and mortality. Changing established and safe techniques would be unwise without good evidence that outcome will be improved.

Disclosure No potential conflicts of interest relevant to this article were reported.

\section{References}

Papers of particular interest, published recently, have been highlighted as:

- Of importance

•- Of major importance

1. Loepke AW, Soriano SG. An assessment of the effects of general anesthetics on developing brain structure and neurocognitive function. Anesth Analg. 2008;106(6):1681-707.

2. Yon JH, Daniel-Johnson J, Carter LB, Jevtovic-Todorovic V. Anesthesia induces neuronal cell death in the developing rat brain via the intrinsic and extrinsic apoptotic pathways. Neuroscience. 2005;135(3):815-27.
3. Ikonomidou C, Bosch F, Miksa M, Bittigau P, Vockler J, Dikranian $\mathrm{K}$, et al. Blockade of NMDA receptors and apoptotic neurodegeneration in the developing brain. Science. 1999;283(5398):70-4.

4. Jevtovic-Todorovic V, Hartman RE, Izumi Y, Benshoff ND, Dikranian K, Zorumski CF, et al. Early exposure to common anesthetic agents causes widespread neurodegeneration in the developing rat brain and persistent learning deficits. J Neurosci. 2003;23(3):876-82.

5. Hayashi H, Dikkes P, Soriano SG. Repeated administration of ketamine may lead to neuronal degeneration in the developing rat brain. Paediatr Anaesth. 2002;12(9):770-4.

6. Kodama M, Satoh Y, Otsubo Y, Araki Y, Yonamine R, Masui K, et al. Neonatal desflurane exposure induces more robust neuroapoptosis than do isoflurane and sevoflurane and impairs working memory. Anesthesiology. 2011;115(5):1-13.

7. Istaphanous GK, Howard J, Nan X, Hughes EA, McCann JC, McAuliffe JJ, et al. Comparison of the neuroapoptotic properties of equipotent anesthetic concentrations of desflurane, isoflurane, or sevoflurane in neonatal mice. Anesthesiology. 2011;114(3):578-87. doi:10.1097/ALN.0b013e3182084a70.

8. Liang G, Ward C, Peng J, Zhao Y, Huang B, Wei H. Isoflurane causes greater neurodegeneration than an equivalent exposure of sevoflurane in the developing brain of neonatal mice. Anesthesiology. 2010;112(6):1325-34.

9. Lu Y, Wu X, Dong Y, Xu Z, Zhang Y, Xie Z. Anesthetic sevoflurane causes neurotoxicity differently in neonatal naive and Alzheimer disease transgenic mice. Anesthesiology. 2010;112(6):1404-16.

10. Fredriksson A, Ponten E, Gordh T, Eriksson P. Neonatal exposure to a combination of $N$-methyl-D-aspartate and gamma-aminobutyric acid type A receptor anesthetic agents potentiates apoptotic neurodegeneration and persistent behavioral deficits. Anesthesiology. 2007;107(3):427-36.

11. Stratmann G. Neurotoxicity of anesthetic drugs in the developing brain. Anesth Analg. 2011;113(5):1170-9.

12. Stratmann G, Sall JW, May LD, Bell JS, Magnusson KR, Rau V, et al. Isoflurane differentially affects neurogenesis and long-term neurocognitive function in 60-day-old and 7-day-old rats. Anesthesiology. 2009;110(4):834-48.

13. • Shih J, May LDV, Gonzalez HE, Lee EW, Alvi RS, Sall JW, et al. Delayed environmental enrichment reverses sevoflurane-induced memory impairment in rats. Anesthesiology 2012;116(3):586-602. This paper demonstrates that factors such as environment may be more important than anaesthesia exposure.

14. Slikker W Jr, Zou X, Hotchkiss CE, Divine RL, Sadovova N, Twaddle NC, et al. Ketamine-induced neuronal cell death in the perinatal rhesus monkey. Toxicol Sci. 2007;98(1):145-58.

15. •• Brambrink AM, Evers AS, Avidan MS, Farber NB, Smith DJ, Martin LD, et al. Ketamine-induced neuroapoptosis in the fetal and neonatal rhesus macaque brain. Anesthesiology 2012;116(2): 372-84. This paper demonstrates significant ketamine induced apoptosis in the non human primate.

16. •• Paule MG, Li M, Allen RR, Liu F, Zou X, Hotchkiss C, et al. Ketamine anesthesia during the first week of life can cause longlasting cognitive deficits in rhesus monkeys. Neurotoxicol Teratol. 2011;33(2):220-30. This paper demonstrates that ketamine exposure results in long term cognitive changes in the non-human primate.

17. • Brambrink AM, Evers AS, Avidan MS, Farber NB, Smith DJ, Zhang X, et al. Isoflurane-induced neuroapoptosis in the neonatal rhesus macaque brain. Anesthesiology 2010;112:834-41. This paper demonstrates that ketamine exposure results in long term cognitive changes in the non-human primate.

18. Ma D, Williamson P, Januszewski A, Nogaro M, Hossain M, Ong $\mathrm{L}$, et al. Xenon mitigates isoflurane-induced neuronal apoptosis in the developing rodent brain. Anesthesiology 2007;106(4): $746-53$. 
19. Sanders RD, Xu J, Shu Y, Januszewski A, Halder S, Fidalgo A, et al. Dexmedetomidine attenuates isoflurane-induced neurocognitive impairment in neonatal rats. Anesthesiology. 2009;110(5):1077-85.

20. Niu L, Cao B, Zhu H, Mei B, Wang M, Yang Y, et al. Impaired in vivo synaptic plasticity in dentate gyrus and spatial memory in juvenile rats induced by prenatal morphine exposure. Hippocampus. 2009;19(7):649-57.

21. Head BP, Patel HH, Niesman IR, Drummond JC, Roth DM, Patel $\mathrm{PM}$. Inhibition of $\mathrm{p} 75$ neurotrophin receptor attenuates isofluranemediated neuronal apoptosis in the neonatal central nervous system. Anesthesiology. 2009;110(4):813-25.

22. Wang C, Sadovova N, Hotchkiss C, Fu X, Scallet AC, Patterson TA, et al. Blockade of $N$-methyl-D-aspartate receptors by ketamine produces loss of postnatal day 3 monkey frontal cortical neurons in culture. Toxicol Sci. 2006;91(1):192-201.

23. Braun S, Gaza N, Werdehausen R, Hermanns H, Bauer I, Durieux $\mathrm{ME}$, et al. Ketamine induces apoptosis via the mitochondrial pathway in human lymphocytes and neuronal cells. Br J Anaesth. 2010;105(3):347-54.

24. - Briner A, De Roo M, Dayer A, Muller D, Habre W, Vutskits L. Volatile anesthetics rapidly increase dendritic spine density in the rat medial prefrontal cortex during synaptogenesis. Anesthesiology 2010;112(3):546-56. These papers from Vutskits' group demonstrate how changes occur in dendritic morphology with lower doses and over a longer period of development compared to apoptosis.

25. De Roo M, Klauser P, Briner A, Nikonenko I, Mendez P, Dayer A, et al. Anesthetics rapidly promote synaptogenesis during a critical period of brain development. PLoS ONE. 2009;4(9):e7043.

26. Vutskits L, Gascon E, Tassonyi E, Kiss JZ. Clinically relevant concentrations of propofol but not midazolam alter in vitro dendritic development of isolated gamma-aminobutyric acidpositive interneurons. Anesthesiology. 2005;102(5):970-6.

27. Vutskits L, Gascon E, Tassonyi E, Kiss JZ. Effect of ketamine on dendritic arbor development and survival of immature GABAergic neurons in vitro. Toxicol Sci. 2006;91(2):540-9.

28. Lemkuil BP, Head BP, Pearn ML, Patel HH, Drummond JC, Patel PM. Isoflurane neurotoxicity is mediated by p75NTR-RhoA activation and actin depolymerization. Anesthesiology. 2011; 114(1):49-57. doi:10.1097/ALN.0b013e318201dcb3.

29. Sall JW, Stratmann G, Leong J, McKleroy W, Mason D, Shenoy $\mathrm{S}$, et al. Isoflurane inhibits growth but does not cause cell death in hippocampal neural precursor cells grown in culture. Anesthesiology. 2009;110(4):826-33.

30. Soriano SG, Liu Q, Li J, Liu JR, Han XH, Kanter JL, et al. Ketamine activates cell cycle signaling and apoptosis in the neonatal rat brain. Anesthesiology. 2010;112(5):1155-63.

31. Sanchez V, Feinstein SD, Lunardi N, Joksovic PM, Boscolo A, Todorovic SM, et al. General anesthesia causes long-term impairment of mitochondrial morphogenesis and synaptic transmission in developing rat brain. Anesthesiology 2011;115(5):992-1002.

32. Liu J-R, Liu Q, Li J, Baek C, Han XH, Athiraman U, et al. Noxious stimulation attenuates ketamine-induced neuroapoptosis in the developing rat brain. Anesthesiology. 2012;117(1):64-71. doi:10.1097/ALN.0b013e31825ae693.

33. Bouman NH, Koot HM, Hazebroek FW. Long-term physical, psychological, and social functioning of children with esophageal atresia. J Pediatr Surg. 1999;34(3):399-404.

34. Chacko J, Ford WD, Haslam R. Growth and neurodevelopmental outcome in extremely-low-birth-weight infants after laparotomy. Pediatr Surg Int. 1999;15(7):496-9.

35. Doyle LW, Callanan C, Carse E, Chariton MP, Drew J, Ford G, et al. Surgery and the tiny baby: sensorineural outcome at 5 years of age. J Paediatr Child Health. 1996;32(2):167-72.
36. Laing S, Walker K, Ungerer J, Badawi N, Spence K. Early development of children with major birth defects requiring newborn surgery. J Paediatr Child Health. 2011;47(3):140-7.

37. Walker K, Halliday R, Holland AJ, Karskens C, Badawi N. Early developmental outcome of infants with infantile hypertrophic pyloric stenosis. J Pediatr Surg. 2010;45(12):2369-72.

38. • Flick RP, Katusic SK, Colligan RC, Wilder RT, Voigt RG, Olson $\mathrm{MD}$, et al. Cognitive and behavioral outcomes after early exposure to anesthesia and surgery. Pediatrics 2011;128(5):1053-61. A cohort study that demonstrates an association between repeated exposure and poor neurobehavioral outcome.

39. Flick RP, Lee K, Hofer RE, Beinborn CW, Hambel EM, Klein MK, et al. Neuraxial labor analgesia for vaginal delivery and its effects on childhood learning disabilities. Anesth Analg. 2011;112(6):1424-31.

40. Sprung J, Flick RP, Wilder RT, Katusic SK, Pike TL, Dingli M, et al. Anesthesia for cesarean delivery and learning disabilities in a population-based birth cohort. Anesthesiology. 2009;111(2):302-10.

41. - Wilder RT, Flick RP, Sprung J, Katusic SK, Barbaresi WJ, Mickelson C, et al. Early exposure to anesthesia and learning disabilities in a population-based birth cohort. Anesthesiology 2009;110(4): 796-804. A cohort study that demonstrates an association between repeated exposure and poor neurobehavioral outcome.

42. Sprung J, Flick RP, Katusic SK, Colligan RC, Barbaresi WJ, Bojanic K, et al. Attention-deficit/hyperactivity disorder after early exposure to procedures requiring general anesthesia. Mayo Clin Proc. 2012;87(2):120-9.

43. - Di Maggio C, Sun L, Kakavuoli A, Byrne M, Li G. A retrospective cohort study of the association of anesthesia and hernia repair surgery with behavioral and developmental disorders in young children. J Neurosurg Anesthesiol. 2009;21:286-91. This cohort study demonstrated an association between hernia repair and adverse neurobehavioral outcome.

44. Di Maggio C, Sun L, Li G. Early childhood exposure to anesthesia and risk of developmental and behavioral disorders in a sibling birth cohort. Anesth Analg. 2011;113(5):1143-51.

45. • Hansen TG, Pedersen JK, Henneberg SW, Pedersen DA, Murray JC, Morton NS, et al. Academic performance in adolescence after inguinal hernia repair in infancy: a nationwide cohort study. Anesthesiology 2011;114(5):1076-85. This large cohort study found no evidence for an association between exposure to brief anaesthesia in early childhood and school performance.

46. Block RI, Thomas JJ, Bayman EO, Choi JY, Kimble KK, Todd MM. Are anesthesia and surgery during infancy associated with altered academic performance during childhood? Anesthesiology. 2012;117(3):494-503.

47. $\bullet$ Bartels M, Althoff RR, Boomsma DI. Anesthesia and cognitive performance in children: no evidence for a causal relationship. Twin Res Hum Genet. 2009;12(3):246-53. An important study that found that in twins discordant for anaesthesia exposure there was no increased risk with exposure.

48. Ing C, DiMaggio C, Whitehouse A, Hegarty MK, Brady J, von Ungern-Sternberg BS, et al. Long-term differences in language and cognitive function after childhood exposure to anesthesia. Pediatrics. 2012;130(3):e476-85.

49. Sun LS, Li G, Dimaggio CJ, Byrne MW, Ing C, Miller TL, et al. Feasibility and pilot study of the Pediatric Anesthesia NeuroDevelopment Assessment (PANDA) project. J Neurosurg Anesthesiol. 2012;24(4):382-8.

50. Davidson A, McCann ME, Morton N. Anesthesia neurotoxicity in neonates: the need for clinical research. Anesth Analg. 2007; 105(3):881-2. 